\title{
Evaluation of the discretization in the spectral resolution for the solution of the line-by-line method in problems with participating gases
}

\author{
Aline Ziemniczak ${ }^{1}$ (D) - Felipe Ramos Coelho ${ }^{1}$. Fernando Marcelo Pereira ${ }^{1}$. Paulo Roberto Pagot ${ }^{2}$. \\ Francis Henrique Ramos França ${ }^{1}$
}

Received: 3 May 2019 / Accepted: 31 July 2019 / Published online: 7 August 2019

(c) The Brazilian Society of Mechanical Sciences and Engineering 2019

\begin{abstract}
Despite the complexity to solve problems involving radiation heat transfer in participating media, especially due to the strong spectral dependence of the absorption coefficient, thermal radiation cannot be neglected in several applications, such as in combustion processes. This study proposes modeling of the spectral absorption coefficient by means of line-by-line integration method (LBL), which can take into account in full detail the complex spectral dependence of the absorption coefficient. The HITEMP 2010 database is used to generate the absorption cross-sections. An evaluation as to the spectral resolution for the LBL integration is performed; the results show that, even for a considerably low spectral refinement, the LBL can still provide accurate results. The lower spectral resolutions are obtained through a methodology to reduce the LBL spectral discretization based on a reference spectrum, which contributes significantly to the satisfactory accuracy reported in this study. The analysis is applied to a set of one-dimensional, non-isothermal medium slabs. In this way, the LBL integration gains space to solve more complex engineering problems with viable computational time and may even be a viable alternative to the use of simpler spectral models such as SLW, WSGG, among others.
\end{abstract}

Keywords Thermal radiation $\cdot$ Participating gas model $\cdot$ HITEMP $\cdot$ LBL integration $\cdot$ Spectral resolution

\section{Introduction}

The calculation of thermal radiation in participating gases is by itself a very complex phenomenon, with many intervening factors. One of them is the spectral treatment of the absorption-emission coefficient, which shows a highly complex dependence with the wavenumber, temperature, and gas composition. Furthermore, the non-uniformity in the temperature and participating gases concentration fields makes calculations even more difficult, which strongly affect the spectral absorption coefficient. This is especially critical in combustion applications. Thus, the treatment of the spectral

Technical Editor: Jader Barbosa Jr..

Aline Ziemniczak

aline.ziemniczak@ufrgs.br

1 Department of Mechanical Engineering, Federal University of Rio Grande do Sul, Porto Alegre, Brazil

2 CENPES/PDEP/TPP - PETROBRAS, Petróleo Brasileiro S. A., Rio de Janeiro, Rio de Janeiro, Brazil properties of the gases, or mixture of gases, is a fundamental step to obtain consistent results. This has led to an ever continuous development of gas models to obtain reliable results with a suitable computational time.

Spectral models, for the treatment of thermal radiation, demand access to high-resolution spectral database, such as HITRAN2012 [1] and HITEMP2010 [2]. The accuracy of the solution obtained with the models is directly related to the quality/accuracy of adopted spectral database. The line-by-line integration method (LBL) can be considered the most accurate approach for spectral modeling. The method takes into account the calculation for the absorption coefficient at each wavelength in the integration of the radiative transfer equation.

In this sense, there are studies that make comparisons between the different database in order to evaluate aspects such as the precision and the calculation time. Chu et al. [3] investigated the effects of using multiple spectral databases: HITEMP1995, HITRAN2004, HITRAN2008, HITEMP2010, and updated CDSD-1000, for the solution of the LBL integration in one-dimensional enclosure between 
two parallel plates filled with real gases, for a $\mathrm{CO}_{2}, \mathrm{H}_{2} \mathrm{O}$ or their mixtures. The results for the different databases showed that the computation of the radiative transfer in general presents strong dependence with the used database. Significant differences were found between the results of the LBL method using HITEMP2010 for those using older databases, so the authors recommended applying the HITEMP2010 database as a benchmark solution to evaluate the accuracy of other approximate models.

Multiple studies have made use of the HITEMP2010 spectral database to obtain results for non-gray gas models, such as the statistical narrow band (SNB) model (Rivière and Soufiani [4], Chu et al. [3]), the spectral-line-based weighted-sum-of-gray-gases (SLW) model (Pearson et al. [5-7] and Liu et al. [8]), the full-spectrum k-distribution (FSK) model (Modest et al. [9, 10] and Cai and Modest [11]), and to obtain new correlations for the weighted-sumof-gray-gases model (Kangwanpongpan et al. [12]; Dorigon et al. [13]; Cassol et al. [14]; Bordbar et al. [15]; Guo et al. [16]; Brittes et al. [17]; Centeno et al. [18]). There is a consensus in the literature $[3,19,20]$ that LBL integration can be taken as the benchmark solution against which all the other models should be compared. There are a few requirements though to be met. The LBL solution should be built on adequate spectral database and involves sufficient spectral resolution discretization. This can restrict the method to simpler, uncoupled problems.

In recent works, Chu et al. [21] used HITEMP2010 and CDSD-1000 databases to obtain the LBL results that serve as benchmark solution to evaluate the accuracy of other models. The LBL, SNB, SNBCK, WSGG, and FSCK models were used in the assessment on the problem that investigated the effects of total pressure on gas radiation heat transfer in 1D parallel plate geometry. Chu et al. [22] evaluated the spectral line resolution for the LBL model obtained with HITEMP2010 database in one and two-dimensional enclosures containing $\mathrm{H}_{2} \mathrm{O} / \mathrm{CO}_{2} / \mathrm{N}_{2}$. The results showed that spectral line resolution, which varied from 0.02 to $0.08 \mathrm{~cm}^{-1}$, might have a significant impact on computing time.

Despite the considerable progress of the spectral models in the last decades, there are some gaps in this area of knowledge, and therefore, there is still ample space for improvements of gas models to lead to accurate solutions with efficient computational time, especially for application in which thermal radiation is to be solved together with other phenomena. One noteworthy example is flames, which also involve modeling of turbulent fluid flow and chemical kinetics.

This paper presents solutions of radiation heat transfer in non-isothermal homogeneous gas mixtures using the LBL integration with spectral database HITEMP2010 for several discretization values for the absorption cross-section. The solutions for the LBL integration will be compared with the solution established as reference in order to find the optimal discretization. The resolutions lower than the reference are obtained through a methodology which reduces the discretization of the reference spectrum, resulting in higher accuracy than if the spectrum was generated for the lower resolution.

The main goal of the present study is to present an evaluation regarding the dependence of the spectral resolution used for the calculation of absorption coefficients for the LBL integration. In other words, the number of wavenumber intervals is enough for the LBL integration to become reliable. This evaluation may indicate whether the simulations with larger sets of wavenumber values are really indispensable in complex analysis, such as real operations conditions in combustion problems. By optimizing the spectral resolution in the LBL integration, an aspect little addressed in the literature, the method can be applied to more complex physical configurations to evaluate other gas models.

\section{Spectral modeling and the spectral absorption coefficient}

The calculation for the absorption cross-section depends on the modeling of spectral lines, which form the spectrum of radiation. A wavenumber (or a determined frequency) is associated with a transition from one energy level to another (vibration-rotation). In the absence of other effects, this spectrum would be composed of infinitesimal thickness spectral lines positioned at certain wavenumbers associated with each molecular or atomic discrete energy transition. In the case of radiation from participating gases in most engineering applications, with temperatures up to $2500 \mathrm{~K}$, collisions between the molecules are the main causes of spectral line broadening. According to Siegel and Howell [23], when collision broadening is dominant, the Lorentz profile can be used in the determination of the absorption cross-section. It is given by:

$C_{\eta}=\sum_{i} \frac{S_{i}}{\pi} \frac{\gamma_{i}}{\gamma_{i}^{2}+\left(\eta-\eta_{i}\right)^{2}}$

where $C_{\eta}$ is the absorption cross-section, in units $\mathrm{cm}^{2} / \mathrm{mol}, S_{i}$ is the integrated line intensity $\mathrm{cm} / \mathrm{mol}, \eta_{i}$ is the line location, in $\mathrm{cm}^{-1}$, and $\gamma_{i}$ is the half-width, in $\mathrm{cm}^{-1}$. The half-width is calculated by:

$\gamma_{i}=\left(\frac{T_{\mathrm{ref}}}{T}\right)^{n} p_{s} \gamma_{\mathrm{self}, i}+\left(1-p_{s}\right) \gamma_{\mathrm{air}, i}$

where $p_{s}$ is the partial pressure, in atm, $T$ is the temperature, in $\mathrm{K}, T_{\text {ref }}$ is the reference temperature $(296 \mathrm{~K}), \gamma_{\text {self }}$ is the line self-broadening, $\gamma_{\text {air }}$ is the broadening caused by the air, both 
in $\mathrm{cm}^{-1}$ atm $^{-1}$, and $n$ is the temperature dependence coefficient. The parameters $n, \gamma_{\text {self }}$ and $\gamma_{\text {air }}$ are provided by the HITEMP database [2].

The integrated line intensity $S_{i}$ in the HITEMP2010 is obtained at the temperature of $1000 \mathrm{~K}$, but is converted to a temperature of $296 \mathrm{~K}$ in its compilation, which is the reference temperature in HITRAN database series. When using the HITEMP2010, it is needed to convert $S_{i}$ to the desired temperature. According to Rothman et al. [2], the equation below is used:

$S_{i}(T)=S_{i}\left(T_{\text {ref }}\right) \frac{Q\left(T_{\text {ref }}\right)}{Q(T)} \frac{\exp \left(-C_{2} E_{i} / T\right)}{\exp \left(-C_{2} E_{i} / T_{\text {ref }}\right)} \frac{\left[1-\exp \left(-C_{2} \nu_{i} / T\right)\right]}{\left[1-\exp \left(-C_{2} v_{i} / T_{\text {ref }}\right)\right]}$

where $Q$ is the total internal partition sums, dimensionless, $v_{i}$ is the energy difference between the initial end final state, in $\mathrm{cm}^{-1}, E_{i}$ is the energy of the lower state, also in $\mathrm{cm}^{-1}$, and $C_{2}$ is the is the second Planck's constant equal to $0.0143877 \mathrm{~m} \mathrm{~K}$.

Figure 1 illustrates the irregular behavior of the absorption cross-section for the species of $\mathrm{CO}_{2}$ and $\mathrm{H}_{2} \mathrm{O}$. The spectra were obtained at the temperature of $1000 \mathrm{~K}$ and for the partial pressure of $0.1 \mathrm{~atm}$, considering a total pressure of $1 \mathrm{~atm}$ from the HITEMP2010.

Once the absorption cross-sections are calculated, it is possible to obtain the pressure absorption coefficient, from the following equation:

$\kappa_{p, \eta}=N C_{\eta}$

where $N$ is the Loschmidt number, in units of molecule/ $\left(\mathrm{cm}^{3} \mathrm{~atm}\right)$, and $\kappa_{p \eta}$ is the pressure absorption coefficient, in $\mathrm{cm}^{-1} \mathrm{~atm}^{-1}$. The Loschmidt number is then given by:

$N=A\left(\frac{T_{\text {ref }}}{T}\right)$ where $A=2.478 \times 10^{19}$ molecules $/\left(\mathrm{cm}^{3}\right.$ atm $)$, and $T_{\text {ref }}=296 \mathrm{~K}$.

In order to obtain the pressure absorption coefficient $\kappa_{p, \eta}, \mathrm{cm}^{-1}$ atm $^{-1}$, Eqs. (1) to (5) are used. For this study, the medium is composed by a mixture of water vapor and carbon dioxide. The absorption coefficient of the mixture, $\kappa_{\eta \text {,mix }}$, is determined by a simple sum of the absorption coefficients of each participating species, that is,

$\kappa_{\eta, \text { mix }}=\kappa_{\eta, \mathrm{CO}_{2}}+\kappa_{\eta, \mathrm{H}_{2} \mathrm{O}}$

Alternatively, since the absorption coefficient can be calculated by the product of the spectral pressure absorption coefficient and the partial pressure, Eq. (6) can be rewritten as:

$\kappa_{p, \eta, \text { mix }}=\frac{p_{\mathrm{CO}_{2}} \kappa_{p, \eta, \mathrm{CO}_{2}}+p_{\mathrm{H}_{2} \mathrm{O}} \kappa_{p, \eta, \mathrm{H}_{2} \mathrm{O}}}{p}$

where $p=p_{\mathrm{CO}_{2}}+p_{\mathrm{H}_{2} \mathrm{O}}$ is the total pressure of the mixture; $p_{\mathrm{CO}_{2}}$ and $p_{\mathrm{H}_{2} \mathrm{O}}$ are the partial pressures of $\mathrm{CO}_{2}$ and $\mathrm{H}_{2} \mathrm{O}$, respectively; $\kappa_{\eta, \mathrm{CO}_{2}}$ and $\kappa_{\eta, \mathrm{H}_{2} \mathrm{O}}$ are the absorption coefficients, in $\mathrm{cm}^{-1}$, of $\mathrm{CO}_{2}$ and $\mathrm{H}_{2} \mathrm{O}$, respectively.

\subsection{Line-by-line (LBL) integration}

The radiative heat transfer is governed by the radiative transfer equation (RTE), which quantifies the variation in the spectral radiation intensity over a path in the medium. For non-scattering media, it is given by

$\frac{\mathrm{d} I_{\eta}(s)}{\mathrm{d} s}=-\kappa_{\eta, \text { mix }}(s) I_{\eta}(s)+\kappa_{\eta, \text { mix }}(s) I_{\eta b}(s)$

where $I_{\eta}$ is the spectral intensity, $I_{\eta b}$ is the blackbody spectral intensity, both at a local position $s$ along a given path, and $\kappa_{\eta \text {,mix }}$ is the spectral absorption coefficient for the gas

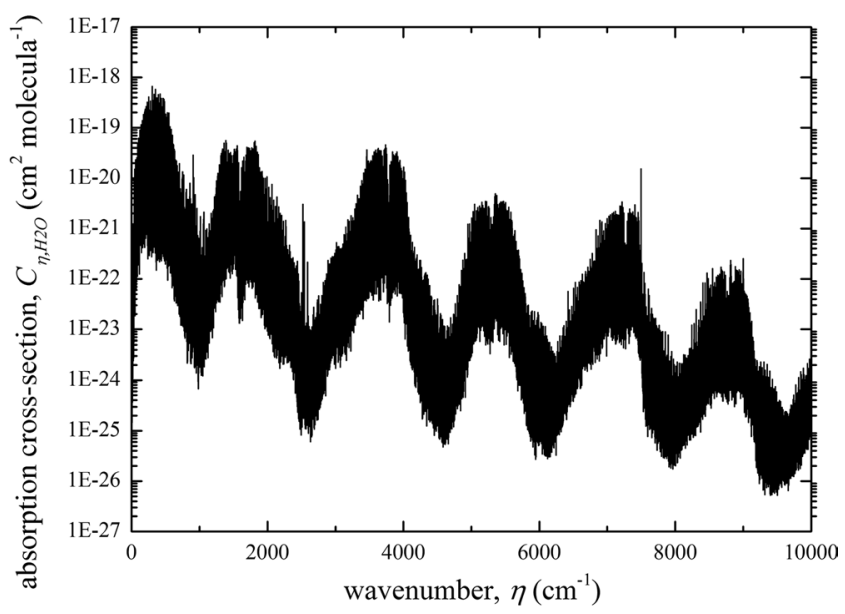

(b)

Fig. 1 Spectral absorption cross-section for: $\mathbf{a ~ C O}_{2}, \mathbf{b ~ H} \mathrm{H}_{2} \mathrm{O}$ at $1000 \mathrm{~K}$ and $1.0 \mathrm{~atm}$ in the full spectral range of $0 \mathrm{~cm}^{-1}<\eta<10000 \mathrm{~cm}^{-1}$ 
mixture, as previously defined. The negative term represents the attenuation on the intensity by the medium absorption, while the positive term corresponds to the increase in the intensity by the medium emission.

The global solution of the RTE requires different types of integration: spectral and spatial. The spatial integration is carried out here by the discrete ordinates method (DOM), which allows the transformation of the RTE into a set of simultaneous partial differential equations. In this method, the RTE is solved for a set of discrete directions covering the total solid angle range of $4 \pi$.

The integral over the solid angle is approximated by a numerical quadrature scheme, in which the equations are solved for a series of $L$ directions. The discrete ordinates method was set for $L=30$ directions, using a Gauss-Legendre quadrature. The RTE, shown in the framework of the discrete ordinates method, can be written for positive and negative directions as:

$\mu_{l} \frac{\mathrm{d} I_{\eta, l}^{+}}{\mathrm{d} x}=-\kappa_{\eta, m}(x) I_{\eta, l}^{+}(x)+\kappa_{\eta, m}(x) I_{\eta b}(x)$

$-\mu_{l} \frac{\mathrm{d} I_{\eta, l}^{-}}{\mathrm{d} x}=-\kappa_{\eta, m}(x) I_{\eta, l}^{-}(x)+\kappa_{\eta, m}(x) I_{\eta b}(x)$

where $\mu_{l}$ is the directional cosine toward $l$ direction $(1 \leq l \leq L)$. Figure 2 shows a schematic representation for a one-dimensional medium slab, configuration that will be used in the present study, and the directions $l$ and positions $x$ for the positive and negative intensities. The medium is considered here to be bounded by black walls, so the boundary conditions for Eqs. (9a) and (9b) are

$I_{\eta, l}^{+}(x=0)=I_{\eta b}(x=0)$

and

$I_{\eta, l}^{-}(x=X)=I_{\eta b}(x=X)$

Once the intensities are solved for each discrete position $x$ and direction $l$, the volumetric radiative heat source (W/

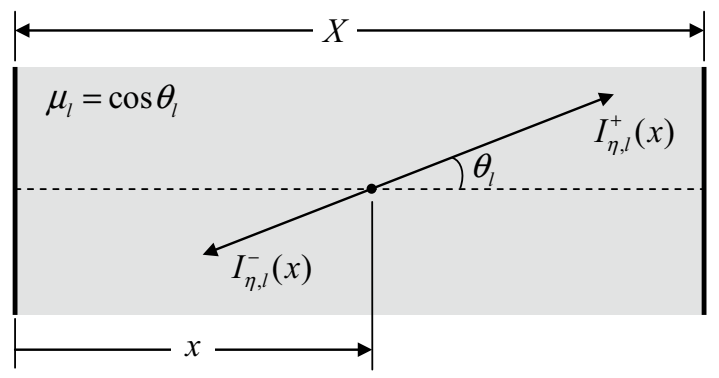

Fig. 2 a Schematic representation of the one-dimensional domain $\left.\mathrm{m}^{3}\right)$ and the radiative heat flux $\left(\mathrm{W} / \mathrm{m}^{2}\right)$ can be determined, respectively, by:

$\dot{q}_{R}(x)=\sum_{l=1}^{L} \int_{\eta}\left\{2 \pi \kappa_{\eta, m}(x) w_{l}\left[I_{\eta, l}^{+}(x)+I_{\eta, l}^{-}(x)\right]-4 \pi \kappa_{\eta, m}(x) I_{\eta b}(x)\right\} \Delta_{\eta}$

$q_{R}^{\prime \prime}(x)=\sum_{l=1}^{L} \int_{\eta} 2 \pi w_{l} \mu_{l}\left[I_{\eta, l}^{+}(x)+I_{\eta, l}^{-}(x)\right] \Delta_{\eta}$

From Eqs. (11) and (12), it follows that the volumetric radiative heat source corresponds to the divergence of the radiative heat flux but with opposite sign, that is, $\dot{q}_{R}(s)=-\mathrm{d} q_{R}^{\prime \prime}(s) / \mathrm{d} x$, which also rises from the application of the radiative energy balance in the medium.

The LBL integration solves the transport equation considering the variation of the absorption coefficient in each wavelength for different temperatures and concentrations for the participant gases. To achieve this, the knowledge of the local spectral absorption coefficients of the species that form the mixture $\mathrm{H}_{2} \mathrm{O}$ and $\mathrm{CO}_{2}$ is necessary. Once the data are available, the LBL integration can provide precise results and is used as a benchmark solution in the spectral validation of gas models.

Currently, the spectral absorption cross-section is generated from databases such as the HITRAN and HITEMP. These databases contain information about parameters related to molecules, in function of the wavenumber. For combustion studies, it is more appropriate to use the HITEMP database, because the data are obtained for high temperatures around $1000 \mathrm{~K}$ and $1500 \mathrm{~K}$. This means that extrapolations to higher temperatures with this database are more appropriate than with HITRAN, which is obtained at the reference temperature of $296 \mathrm{~K}$.

In this study, the spectral absorption coefficients are generated with the HITEMP2010 database for a range of wavenumber between $\eta=0$ and $10,000 \mathrm{~cm}^{-1}$, which comprises the most significant region for thermal radiation in processes of interest in engineering. It is set, as reference, a spectral resolution of $\Delta \eta=0.067 \mathrm{~cm}^{-1}$, correspondent to 150,000 wavenumber intervals $N P$, which values around the spectral lines were computed from Lorentz profile [23].

An approach for reducing the spectral resolution is used, where new resolutions are obtained from a mathematical artifice, consisting in a arithmetic mean for a set of values of the reference spectrum $\left(\Delta \eta=0.067 \mathrm{~cm}^{-1}\right.$ and $N P=150,000$ wavenumber). The number of intervals $y$ for the average calculation is established by Eq. (13). From this new set of intervals, new values for $C \eta$ parameter, necessary for the LBL calculation, are calculated as Eq. (14).

$y=\frac{N P_{\text {reference }}}{N P_{\text {reduced }}}$ 


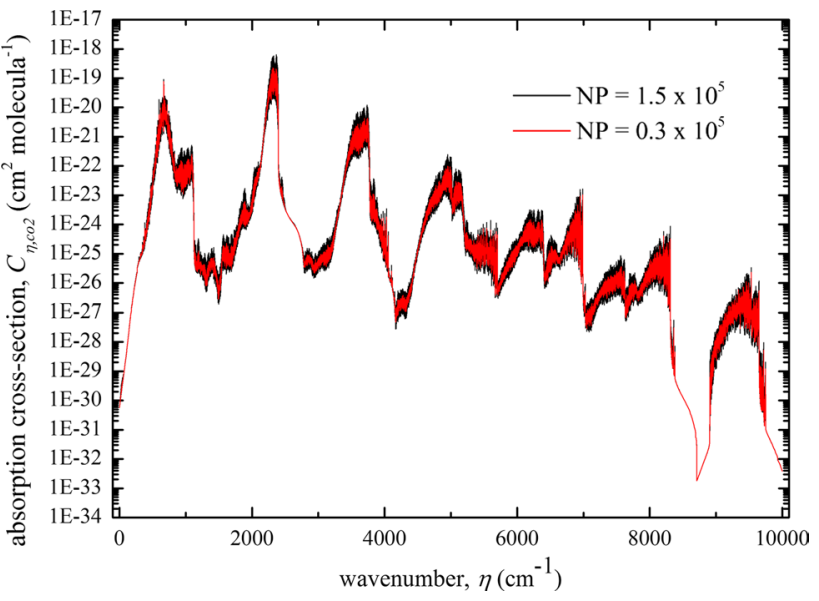

Fig. 3 Spectral absorption cross-section for $\mathrm{CO}_{2}$ at $1000 \mathrm{~K}$ and $1.0 \mathrm{~atm}$ for different spectral resolutions

Table 1 Discretization of spectral resolution with reduction for all the cases for the LBL

\begin{tabular}{ll}
\hline Total of wavenumber $(N P)$ in the spectrum & Spectral resolution $\Delta \eta$ \\
\hline 10,000 & $\Delta_{\eta}=1 \mathrm{~cm}^{-1}$ \\
30,000 & $\Delta_{\eta}=0,33 \mathrm{~cm}^{-1}$ \\
50,000 & $\Delta_{\eta}=0,2 \mathrm{~cm}^{-1}$ \\
80,000 & $\Delta_{\eta}=0,125 \mathrm{~cm}^{-1}$ \\
100,000 & $\Delta_{\eta}=0,1 \mathrm{~cm}^{-1}$ \\
125,000 & $\Delta_{\eta}=0,08 \mathrm{~cm}^{-1}$ \\
150,000 & $\Delta_{\eta}=0,067 \mathrm{~cm}^{-1}$ \\
\hline
\end{tabular}

$C_{\eta_{\text {reduced }}}=\frac{\sum_{i=1}^{y} C_{\eta, i}}{y}$

Figure 3 shows the absorption cross sections for the molecule of $\mathrm{CO}_{2}$ at $1100 \mathrm{~K}$ temperature and for the partial pressure of $0.1 \mathrm{~atm}$ calculated for both the resolution of $\Delta \eta=0.067 \mathrm{~cm}^{-1}$ and for the reduced resolution obtained as $\Delta \eta=0.33 \mathrm{~cm}^{-1}$ corresponding to 30,000 wavenumber intervals. It is possible to observe that the behavior with the reduction is very close to the spectrum considering the reference resolution, only presenting a slight attenuation of peaks and valleys due to the average calculation.

Table 1 specifies the spectral resolution for all the reduced cases tested, obtained from the reference $N P=150,000$ wavenumber intervals.

The spectral database was obtained for a total pressure of $1.0 \mathrm{~atm}$, with gas temperatures ranging from 400 to $2500 \mathrm{~K}$, with interval of $100 \mathrm{~K}$ totalizing 22 temperatures. The absorption coefficients for the gases $\mathrm{H}_{2} \mathrm{O}$ and $\mathrm{CO}_{2}$ $\left(\kappa_{\eta, \mathrm{CO}_{2}}, \kappa_{\eta, \mathrm{H}_{2} \mathrm{O}}\right)$ were obtained for mole fraction of $Y_{\mathrm{H}_{2} \mathrm{O}}=0.1$ and $Y_{\mathrm{CO}_{2}}=0.1$ (therefore, partial pressures of $p_{\mathrm{CO}_{2}}=0.1 \mathrm{~atm}$ and $\left.p_{\mathrm{H}_{2} \mathrm{O}}=0.1 \mathrm{~atm}\right)$. For intermediary temperatures and concentrations, linear interpolations were applied.

\section{Results and discussions}

In this study, the problem of two flat plates with black walls (emissivity of 1.0), which is the preferable geometry to test gas models while keeping the simplicity of the spatial integration. The plates are separated by a distance $X$. Making full advantage of the symmetries raised in this 1D configuration, this partition has proved to lead to sufficiently accurate solution [13, 14, 17]. Three different temperature profiles for non-isothermal media conditions are proposed in the equations below:

$T\left(x^{*}\right)=400 K+(1400 K) \sin ^{2}\left(\pi x^{*}\right)$

$T\left(x^{*}\right)=400 K+(1400 K) \sin ^{2}\left(2 \pi x^{*}\right)$

$T\left(x^{*}\right)=\left\{\begin{array}{l}880 K+(920 K) \sin ^{2}\left(2 \pi x^{*}\right) \\ 400 K+(1400 K)\left\{1-\sin ^{3 / 2}\left[\frac{2}{3} \pi\left(x^{*}-0.25\right)\right]\right\}\end{array}\right.$

where $x^{*}=x / X$ with $X=1.0 \mathrm{~m}$. The proposed temperature profiles range from $400 \mathrm{~K}$, at the walls, to a maximum value of $1800 \mathrm{~K}$. Figure $4 \mathrm{~b}$ shows the temperature profiles analyzed according to Eqs. (15) to (17). A homogeneous condition for the mole fractions of $\mathrm{CO}_{2}$ and $\mathrm{H}_{2} \mathrm{O}$ is considered according to:

$Y_{\mathrm{CO}_{2}}\left(x^{*}\right)=Y_{\mathrm{H}_{2} \mathrm{O}}\left(x^{*}\right)=0,1$

Also, it was tested a case with a non-homogeneous condition for the mole fraction of $\mathrm{CO}_{2}$ given by the following profile (Eq. 19), in combination with the temperature profile given by Eq. 15. The profile is displayed in Fig. $4 \mathrm{~b}$.

$Y_{\mathrm{CO}_{2}}\left(s^{*}\right)=0.2 \sin ^{2}\left(\pi s^{*}\right)$

In this particular test case of non-homogeneous medium, the temperature profile is given by Eq. (15). The profile of the mole fraction of $\mathrm{CO}_{2}$ is displayed in Fig. $4 \mathrm{~b}$.

The solution with a spectral resolution of $\Delta \eta=0.067 \mathrm{~cm}^{-1}$ $(150,000$ wavenumber values) was established as the reference, as already mentioned. The deviations between the different LBL spectral resolutions are calculated in relation to this reference discretization according to:

$\delta=\frac{\left|q_{R, \mathrm{LBL}_{\mathrm{ref}}}^{\prime \prime}-q_{R, \mathrm{LBL}}^{\prime \prime}\right|}{\max \left|q_{R, \mathrm{LBL}_{\mathrm{ref}}}^{\prime \prime}\right|} 100 \%$ 


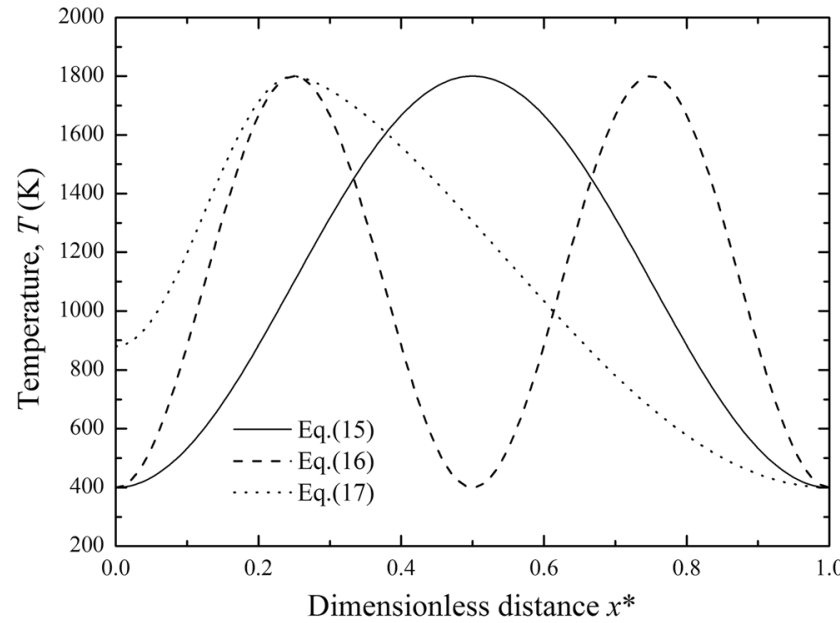

(a)

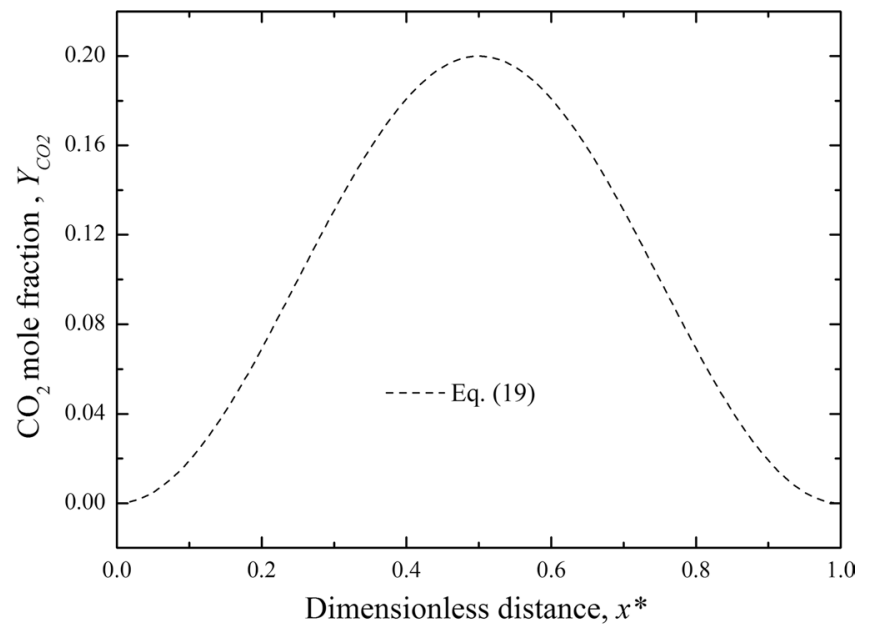

(b)

Fig. 4 aTemperature profiles, $\mathbf{b} \mathrm{CO}_{2}$ mole fraction profile

$\zeta=\frac{\left|\dot{q}_{R, \mathrm{LBL}_{\mathrm{ref}}}-\dot{q}_{R, \mathrm{LBL}}\right|}{\max \left|\dot{q}_{R, \mathrm{LBL}_{\mathrm{ref}}}\right|} 100 \%$

where $\delta$ is the radiative heat deviation, and $\zeta$ is the radiative heat source deviation. The notations $\delta_{\max }$ and $\delta_{\text {avg }}$ will be used for the maximum and average deviations for radiative heat flux. For the radiative heat source, the notations $\zeta_{\max }$ and $\zeta_{\text {avg }}$ will be used for the maximum and average deviations.

Figures 5a, b present the radiative heat flux and source, corresponding to the profile with simple symmetry from

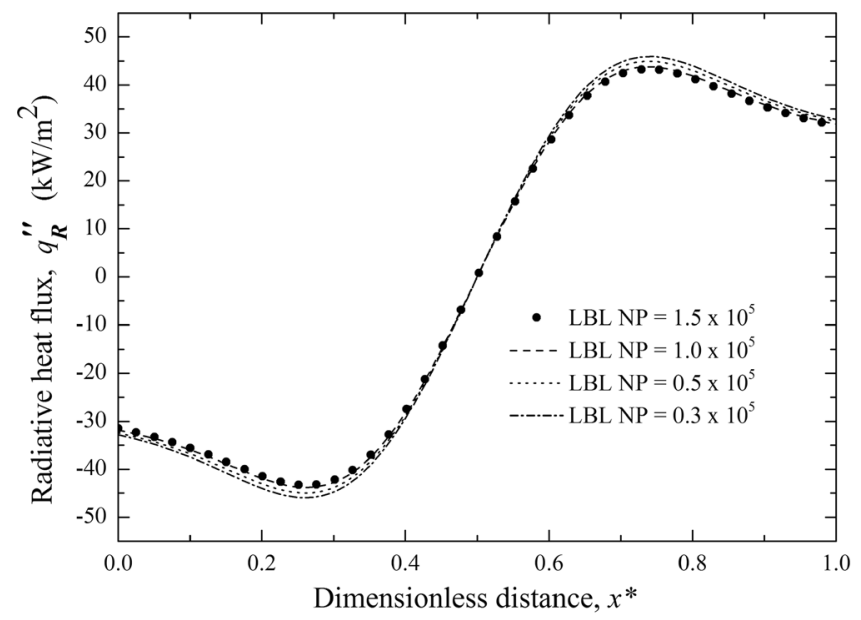

(a)
Eq. (15), for several spectral discretizations. As can be seen, for both the radiative heat flux and volumetric source, the curves for different spectral discretizations showed a behavior very close to each other, where some of the curves practically overlapped. Table 2 shows that the maximum deviation for the less refined spectral discretization $\left(\Delta \eta=0.333 \mathrm{~cm}^{-1}\right)$ presented in Fig. 4, for the radiative heat flux was $\delta_{\max }=6.18 \%$, while, for the radiative heat source, $\zeta_{\max }=4.95 \%$. For the more refined case $\left(\Delta \eta=0.1 \mathrm{~cm}^{-1}\right)$, the maximum deviation for the radiative heat flux was $\delta_{\max }=1.16 \%$, and, for the radiative heat source, $\zeta_{\max }=1.06 \%$. The behavior of the solution for the radiative heat source was expected, with the maximum absolute value in the middle of the domain, where the medium temperature

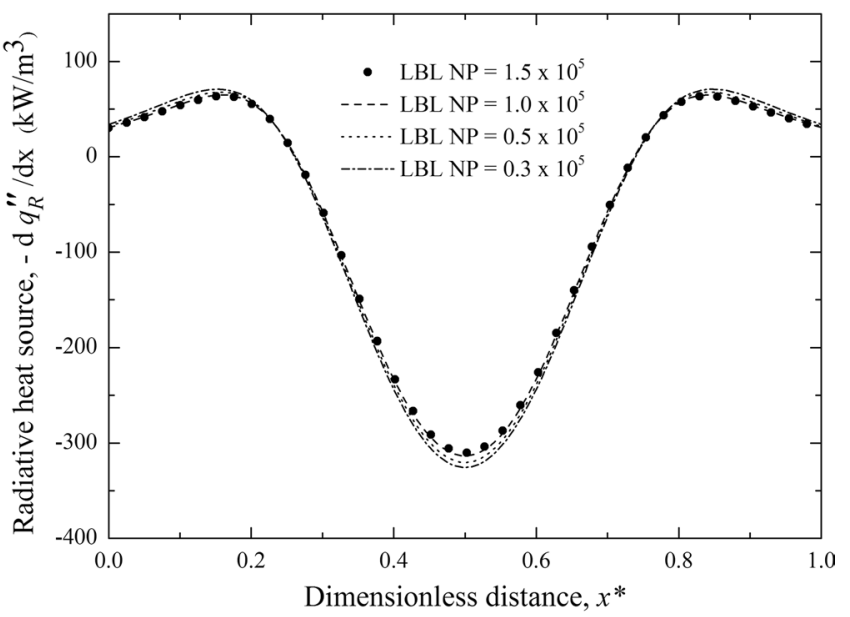

(b)

Fig. 5 Comparison between discretization for the LBL solutions: a radiative heat flux $q_{R}^{\prime \prime}$, and $\mathbf{b}$ radiative heat source $\dot{q}_{R}=-\mathrm{d} q_{R}^{\prime \prime} / \mathrm{d} x$, for temperature profile given by Eq. (15) 
Table 2 Maximum and average deviations between the different discretization for the radiative heat flux, $q_{R}^{\prime \prime}$, and the radiative heat source $\dot{q}_{R}=-\mathrm{d} q_{R}^{\prime \prime} / \mathrm{d} x$ for the profile given by Eq. (15)

\begin{tabular}{lllllll}
\hline$N P$ & $\Delta \eta\left(\mathrm{cm}^{-1}\right)$ & \multicolumn{2}{c}{ Radiative heat flux } & \multicolumn{2}{c}{$\begin{array}{l}\text { Radiative heat } \\
\text { source }\end{array}$} \\
& & $\delta_{\max }$ & $\delta_{\text {avg }}$ & & $\zeta_{\max }$ & $\zeta_{\text {avg }}$ \\
\hline 500,000 & 0.02 & 1.09 & 0.81 & & 0.98 & 0.40 \\
150,000 & 0.067 & - & - & & - \\
125,000 & 0.08 & 0.15 & 0.07 & & 0.07 & 0.04 \\
100,000 & 0.1 & 1.16 & 0.85 & & 1.06 & 0.45 \\
80,000 & 0.125 & 1.73 & 1.25 & & 1.56 & 0.69 \\
50,000 & 0.2 & 3.85 & 2.74 & & 3.26 & 1.57 \\
30,000 & 0.33 & 6.18 & 4.28 & & 4.95 & 2.63 \\
10,000 & 1 & 11.36 & 7.62 & 8.09 & 5.06 \\
\hline
\end{tabular}

is the highest. The negative signal points at the fact that, on balance, the emission from the highest temperature regions in the medium exceeds absorption of the radiation coming from the surroundings, so the radiative heat source becomes negative. On the other hand, the regions in the medium where the temperatures are lower lead to positive radiative heat source, since absorption exceeds emission.

A case considering the same temperature profile with single symmetry, Eq. (15), but with non-homogenous condition for the mole fraction, given by Eq. (19), is presented in Fig. 6a, b for the radiative heat flux and source for different spectral discretizations. The behavior of the solution with non-homogenous concentrations is quite similar to the solution for homogenous condition, where the curves are practically coincident. The deviations in relation to the solution of reference for the radiative heat flux are equal to $\delta_{\max }=2.32 \%$, and radiative heat source equal $\zeta_{\max }=2.51 \%$ for the least refined spectral discretization $\left(\Delta \eta=0.333 \mathrm{~cm}^{-1}\right)$. For the most refined case $\left(\Delta \eta=0.1 \mathrm{~cm}^{-1}\right)$, the maximum deviation for the radiative heat flux was $\delta_{\max }=1.47 \%$, and, for the radiative heat source, $\zeta_{\max }=1.30 \%$, as can be seen in Table 3. Analyzing the results, the inhomogeneity of the mole fraction in the solution does not have a major impact on the deviation of the results relative to the reference solution, where the deviations for the different discretizations are smaller than the results found for the homogeneous cases.

Figure $7 \mathrm{a}, \mathrm{b}$ presents the radiative heat flux and volumetric source, corresponding to the double symmetry temperature profile given by Eq. (16), for several spectral discretizations. A similar behavior to the previous case is observed. Analyzing the different spectral discretizations, once again the curves were very close to each other. The maximum

Table 3 Maximum and average deviations between the different discretization for a non-homogenous case for the radiative heat flux, $q_{R}^{\prime \prime}$, and the radiative heat source $\dot{q}_{R}=-\mathrm{d} q_{R}^{\prime \prime} / \mathrm{d} x$ for temperature and $\mathrm{CO}_{2}$ mole fraction profiles given by Eqs. (15) and (19)

\begin{tabular}{lllllll}
\hline$N P$ & $\Delta \eta\left(\mathrm{cm}^{-1}\right)$ & \multicolumn{2}{c}{ Radiative heat flux } & \multicolumn{2}{c}{$\begin{array}{l}\text { Radiative heat } \\
\text { source }\end{array}$} \\
& & $\delta_{\max }$ & $\delta_{\text {avg }}$ & & $\zeta_{\max }$ & $\zeta_{\text {avg }}$ \\
\hline 150,000 & 0.067 & - & - & - & - \\
125,000 & 0.08 & 1.27 & 0.36 & & 1.11 & 0.86 \\
100,000 & 0.1 & 1.47 & 0.41 & & 1.30 & 1.02 \\
80,000 & 0.125 & 0.07 & 0.03 & & 0.06 & 0.03 \\
50,000 & 0.2 & 1.43 & 0.51 & & 1.48 & 1.08 \\
30,000 & 0.33 & 2.32 & 0.88 & & 2.51 & 1.80 \\
10,000 & 1 & 4.82 & 2.03 & 5.83 & 4.22 \\
\hline
\end{tabular}

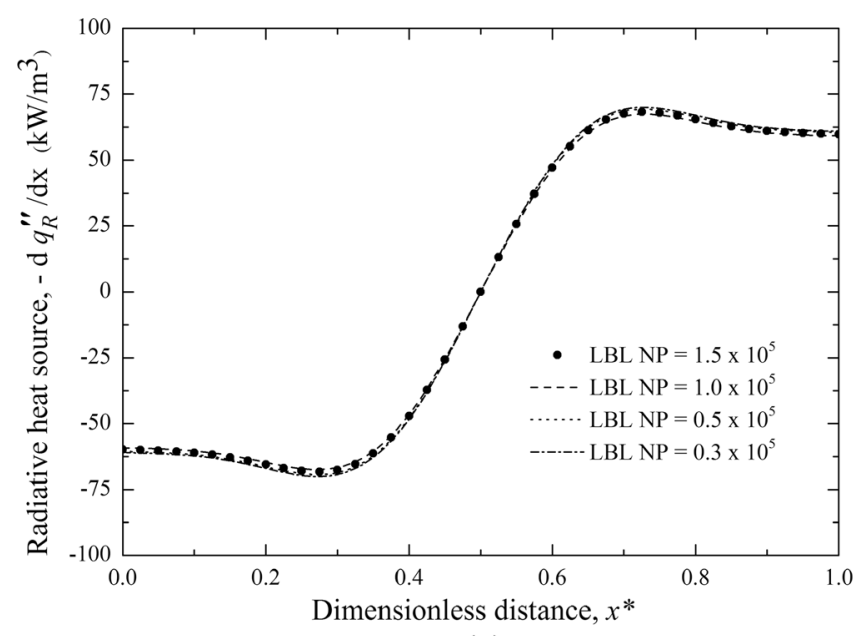

(a)

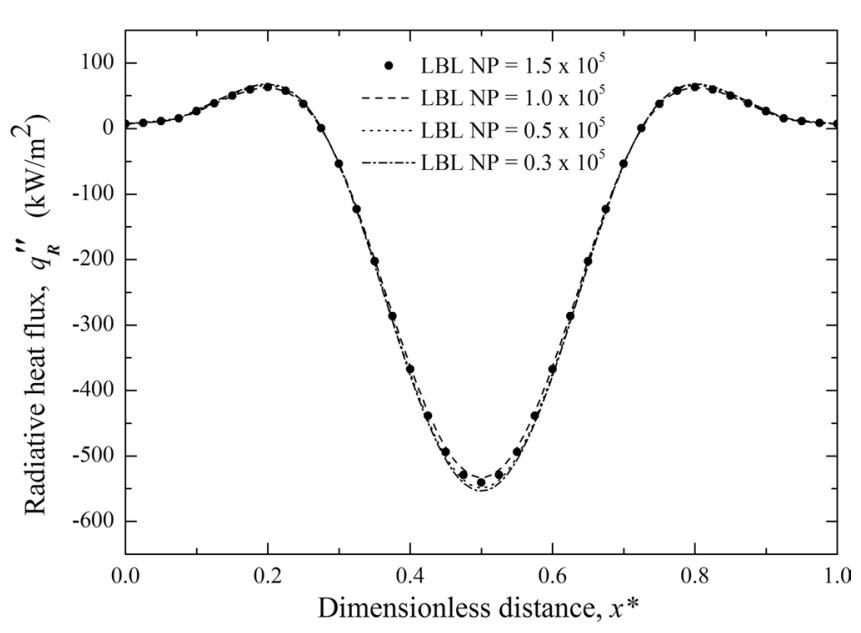

(b)

Fig. 6 Comparison between discretization for the LBL solutions: a radiative heat flux $q_{R}^{\prime \prime}$, and $\mathbf{b}$ radiative heat source $\dot{q}_{R}=-\mathrm{d} q_{R}^{\prime \prime} / \mathrm{d} x$, for temperature and non-homogeneous mole fraction given by Eqs. (15) and (19) 


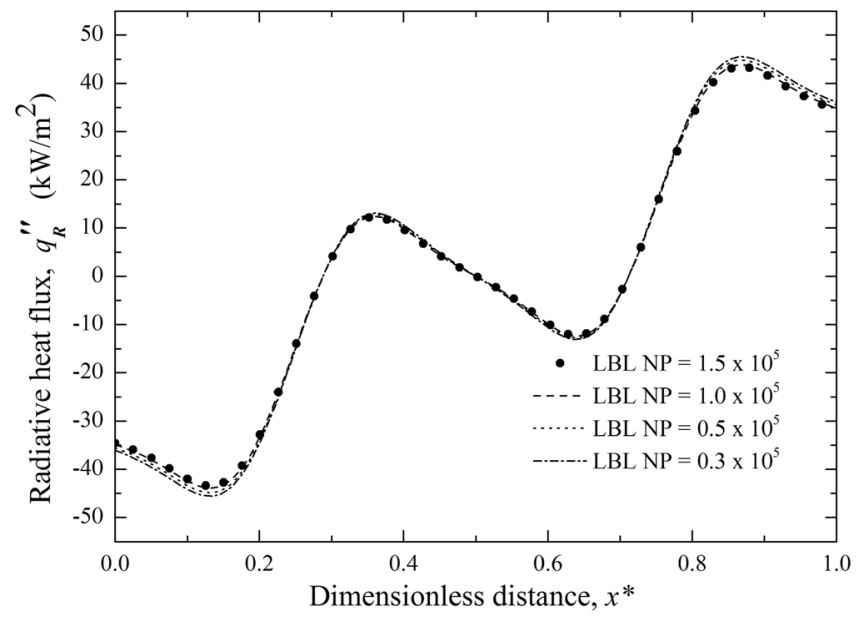

(a)

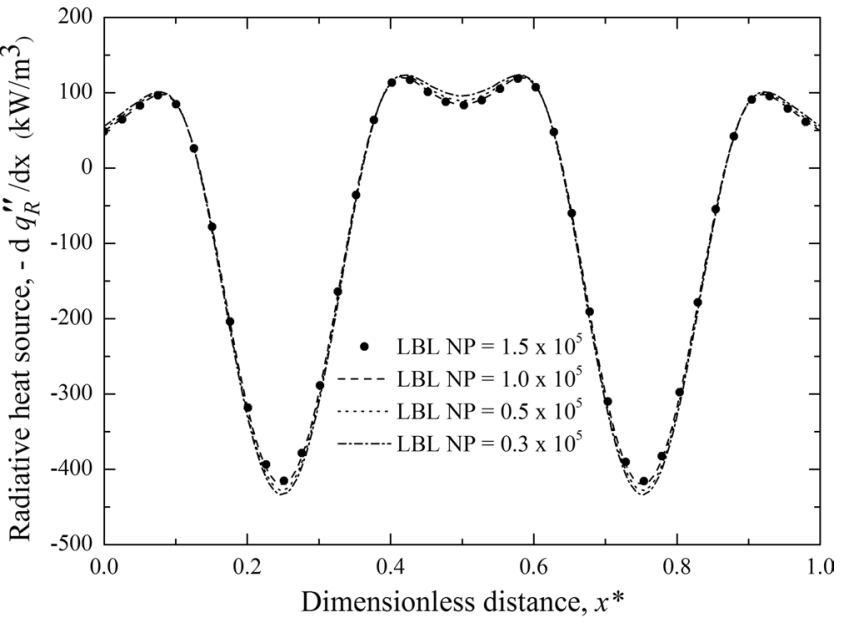

(b)

Fig. 7 Comparison between discretization for the LBL solutions: a radiative heat flux $q_{R}^{\prime \prime}$, and $\mathbf{b}$ radiative heat source $\dot{q}_{R}=-\mathrm{d} q_{R}^{\prime \prime} / \mathrm{d} x$, for temperature profile given by Eq. (16)

Table 4 Maximum and average deviations between the different discretization for the radiative heat flux, $q_{R}^{\prime \prime}$, and the radiative heat source $\dot{q}_{R}=-\mathrm{d} q_{R}^{\prime \prime} / \mathrm{d} x$ for the profile given by Eq. (16)

\begin{tabular}{lllllll}
\hline$N P$ & $\Delta \eta\left(\mathrm{cm}^{-1}\right)$ & \multicolumn{2}{c}{ Radiative heat flux } & \multicolumn{2}{c}{$\begin{array}{l}\text { Radiative heat } \\
\text { source }\end{array}$} \\
& & $\delta_{\max }$ & $\delta_{\text {avg }}$ & & $\zeta_{\max }$ & $\zeta_{\text {avg }}$ \\
\hline 500,000 & 0.02 & 0.97 & 0.51 & & 0.98 & 0.35 \\
150,000 & 0.067 & - & - & & - & - \\
125,000 & 0.08 & 0.14 & 0.06 & & 0.08 & 0.04 \\
100,000 & 0.1 & 1.03 & 0.54 & & 1.00 & 0.41 \\
80,000 & 0.125 & 1.51 & 0.79 & & 1.43 & 0.63 \\
50,000 & 0.2 & 3.21 & 1.70 & & 2.90 & 1.34 \\
30,000 & 0.33 & 4.94 & 2.64 & & 4.29 & 2.22 \\
10,000 & 1 & 8.59 & 4.63 & & 7.28 & 4.11 \\
\hline
\end{tabular}

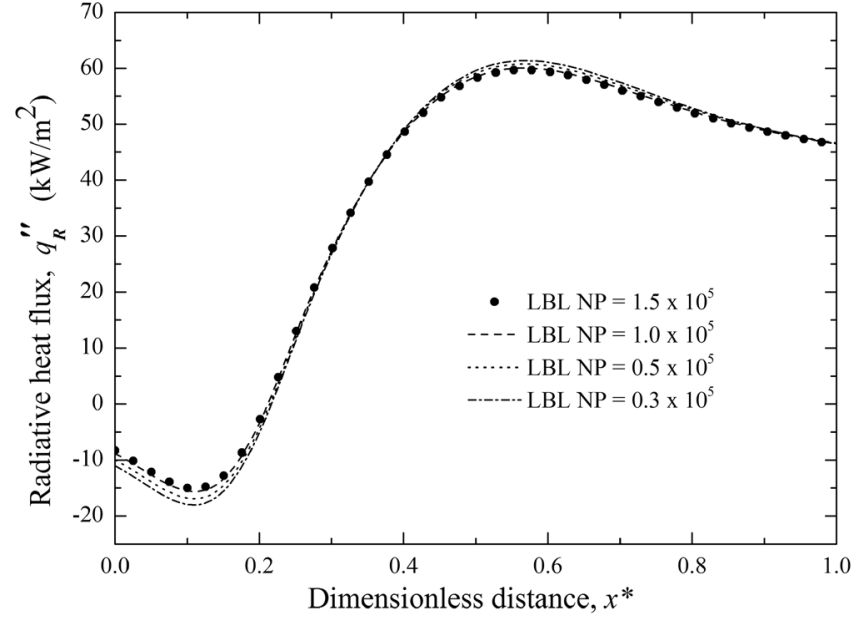

(a) value found for the heat flux and source deviation, in relation to the reference solution, was less than $5 \%$, while the average deviation was less than $2.5 \%$, as can be verified in Table 4 .

Figure $8 \mathrm{a}, \mathrm{b}$ shows the radiative heat flux and radiative heat source corresponding to the temperature profile given by Eq. (17). In this non-symmetric profile, a maximum absolute value of the volumetric heat source takes place in the position where the temperature of the medium is higher, in the same way as for the previous profiles. In this case, the spectral discretization for the LBL $\Delta \eta=0.33 \mathrm{~cm}^{-1}$ showed average deviation of $2.21 \%$ in relation to the reference solution $\left(\Delta \eta=0.067 \mathrm{~cm}^{-1}\right)$, while $\Delta \eta=0.1 \mathrm{~cm}^{-1}$ to an average deviation of $0.345 \%$.

Table 2 shows the values of maximum and average deviations for the radiative heat flux and the volumetric source

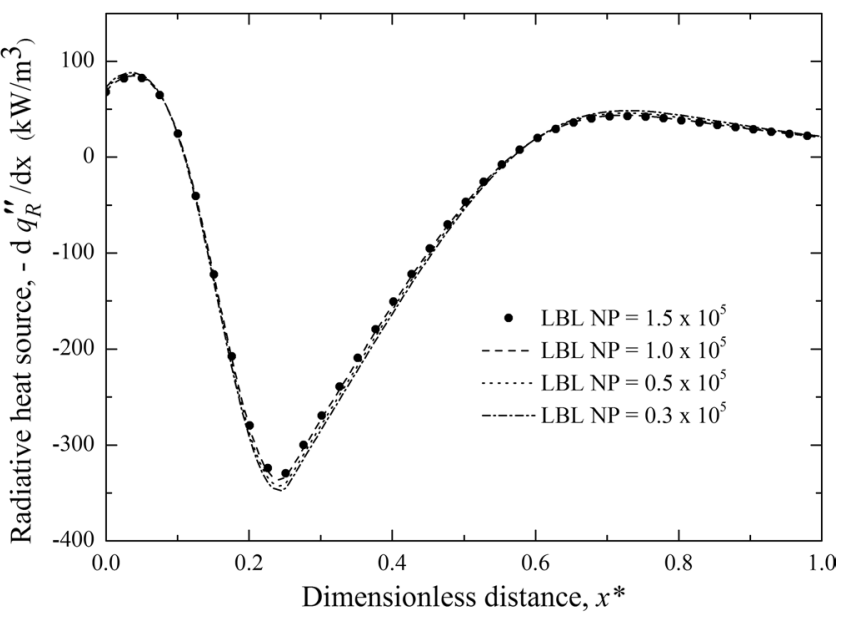

(b)

Fig. 8 Comparison between discretization for the LBL solutions: a radiative heat flux $q_{R}^{\prime \prime}$, and $\mathbf{b}$ radiative heat source $\dot{q}_{R}=-\mathrm{d} q_{R}^{\prime \prime} / \mathrm{d} x$, for temperature profile given by Eq. (17) 
Table 5 Maximum and average deviations between the different discretization for the radiative heat flux, $q_{R}^{\prime \prime}$, and the radiative heat source $\dot{q}_{R}=-\mathrm{d} q_{R}^{\prime \prime} / \mathrm{d} x$ for the profile given by Eq. (17)

\begin{tabular}{lllllll}
\hline$N P$ & $\Delta \eta\left(\mathrm{cm}^{-1}\right)$ & \multicolumn{2}{c}{ Radiative heat flux } & \multicolumn{2}{l}{$\begin{array}{l}\text { Radiative heat } \\
\text { source }\end{array}$} \\
& & $\delta_{\max }$ & $\delta_{\text {avg }}$ & & $\zeta_{\max }$ & $\zeta_{\text {avg }}$ \\
\hline 500,000 & 0.02 & 0.82 & 0.43 & & 1.00 & 0.30 \\
150,000 & 0.067 & - & - & & - \\
125,000 & 0.08 & 0.08 & 0.04 & & 0.07 & 0.03 \\
100,000 & 0.1 & 0.91 & 0.45 & & 1.02 & 0.34 \\
80,000 & 0.125 & 1.36 & 0.66 & & 1.47 & 0.51 \\
50,000 & 0.2 & 3.04 & 1.43 & & 3.05 & 1.15 \\
30,000 & 0.33 & 4.92 & 2.21 & & 4.55 & 1.92 \\
10,000 & 1 & 9.08 & 3.89 & 7.23 & 3.69 \\
\hline
\end{tabular}

term for the symmetric temperature profile (Eq. 15), Table 4 for the temperature profile with double symmetry (Eq. 16), and Table 5 for the asymmetric temperature profile (Eq. 17), all considering the different spectral discretizations. As mentioned before, the solution with $\Delta \eta=0.067 \mathrm{~cm}^{-1}$ was established as a reference for the comparative analysis of the results. It is possible to observe that the highest deviation occurs for the case with the less refined spectral discretization $\Delta \eta=1 \mathrm{~cm}^{-1}$; however, despite the deviations found, the solution could still be considered satisfactory in case of the considerable reduction in the computational time. It is also worth mentioning that, for $\Delta \eta=0.125 \mathrm{~cm}^{-1}$, the deviations between the solutions are practically negligible, less than $1 \%$ for the maximum deviations and $0.5 \%$ for the mean deviations.

A database for a larger spectral resolution $\Delta \eta=0.02 \mathrm{~cm}^{-1}$ corresponding to a $N P=500,000$ was generated with the purpose of showing that the solution with the resolution set as reference, $N P=150,000$, is already satisfactory. Figures 9 and 10 show a comparison of the radiative heat flux

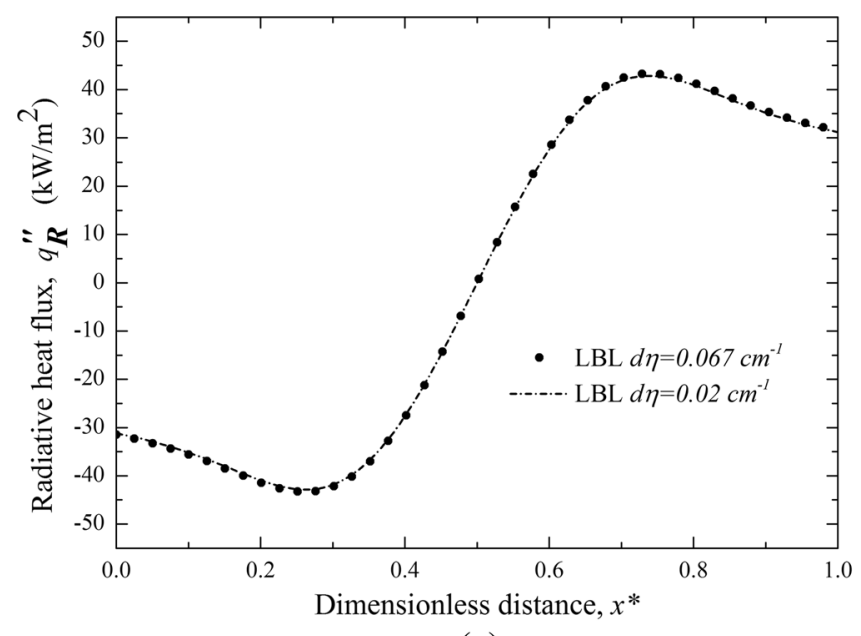

(a)

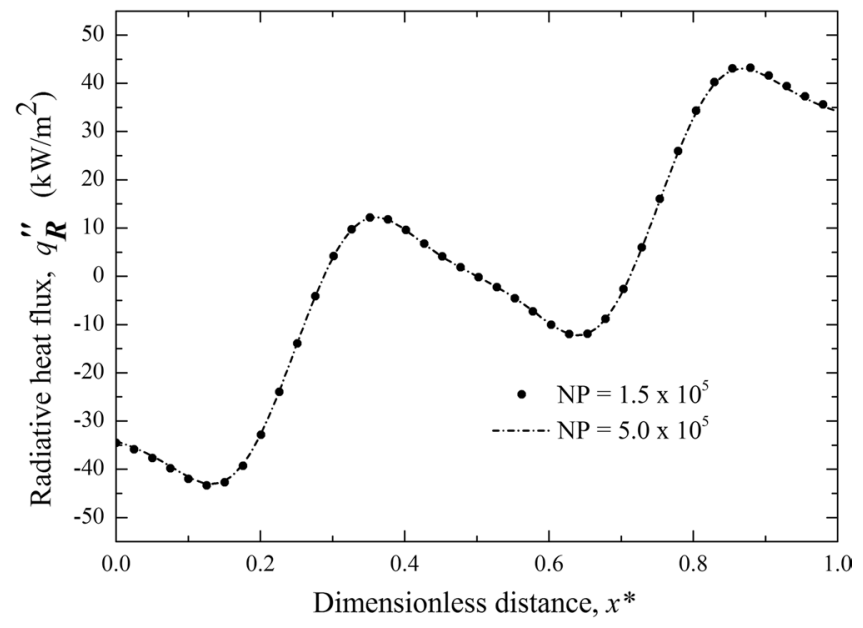

(b)

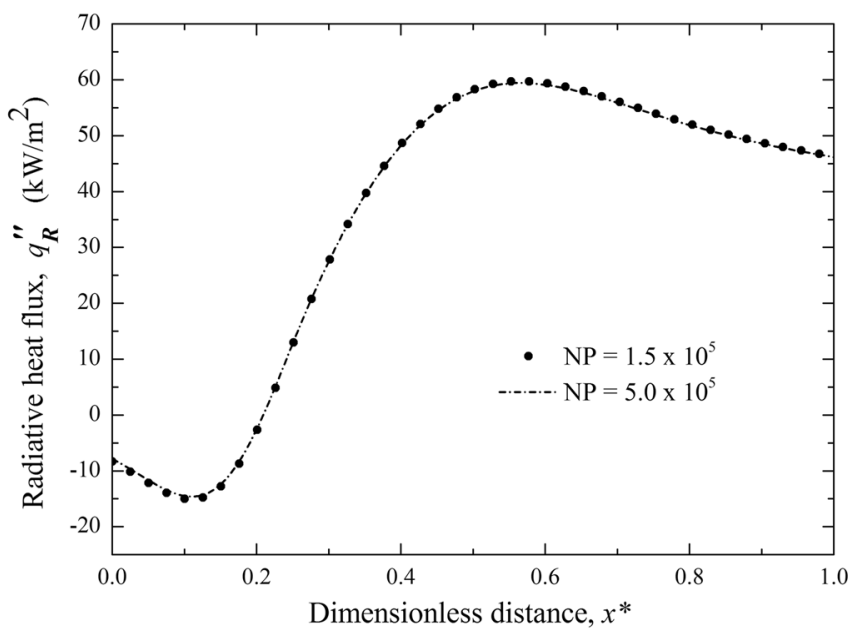

(c)

Fig. 9 Radiative heat flux, $q_{R}^{\prime \prime}$, for the three temperature profiles given by: a Eq. (15), b Eq. (16) and c Eq. (17) 


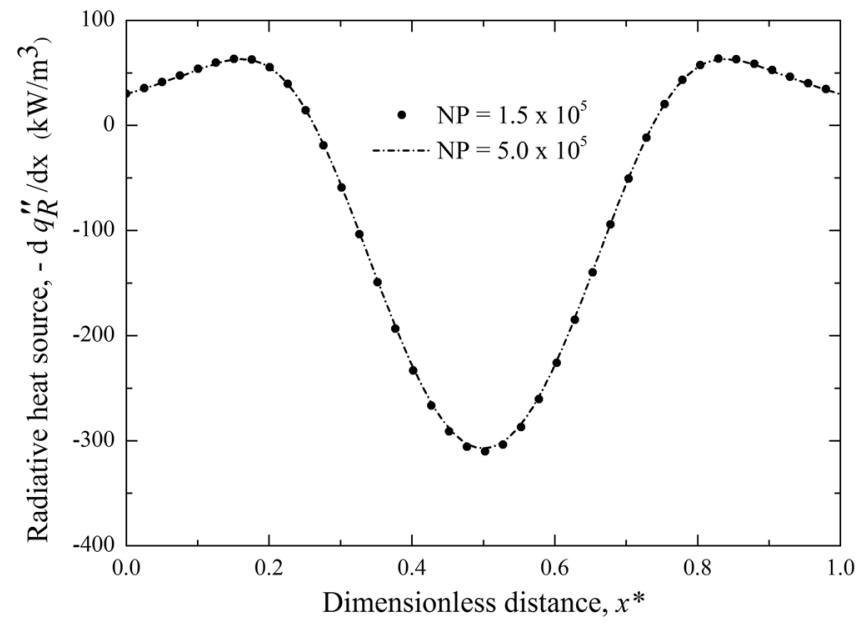

(a)

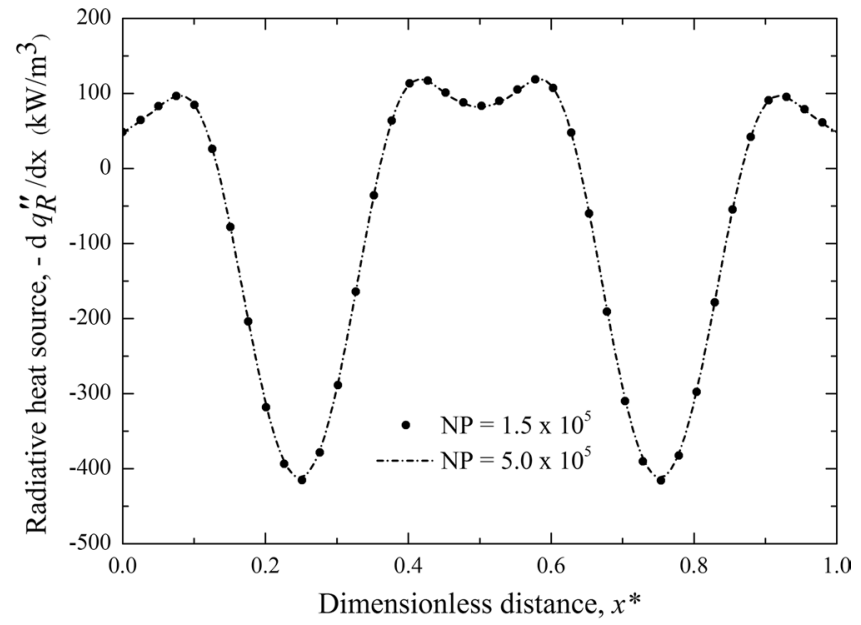

(b)

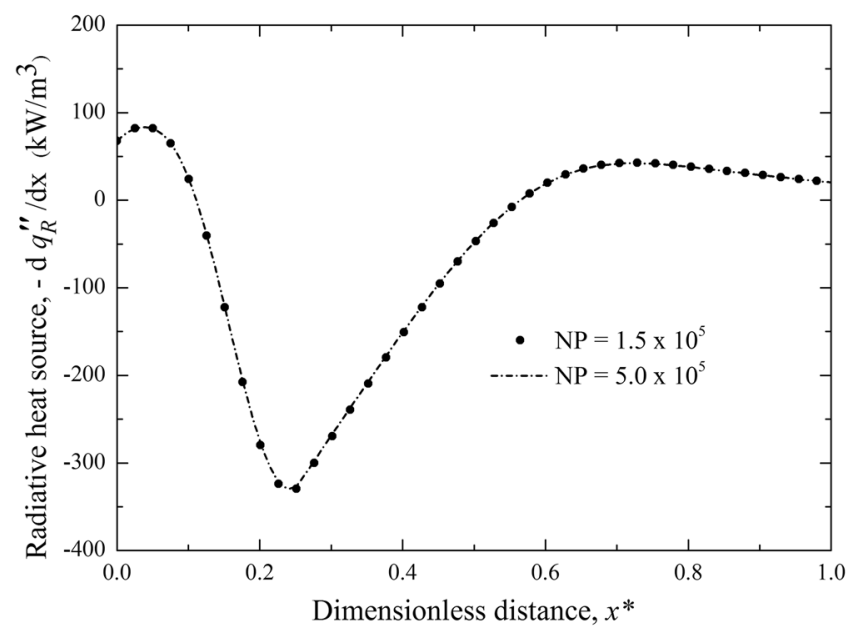

(c)

Fig. 10 Radiative heat source, $q_{R}^{\prime \prime}$, for the three temperature profiles given by: a Eq. (15), b Eq. (16) and c Eq. (17)

and radiative heat source for the three temperature profiles studied. It is possible to observe that the results with $N P=150,000$ and $N P=500,000$ do not show significant variation between solutions, which corroborates the assertion that a solution with $N P=150,000$ already provide satisfactory results. The maximum deviation value found between both resolutions is less than $1 \%$, a difference that does not justify the computational effort expended for the solution with higher spectral resolution.

\section{Conclusions}

This paper showed an evaluation of the spectral discretization for the solution of radiation problems solved by the LBL integration using HITEMP2010 spectral database. Results for three different temperature profiles were presented. Several spectral resolutions were tested in order to evaluate the real necessity of such discretizations on the final results in problems solved with LBL integration. A solution with a spectral resolution of $0.067 \mathrm{~cm}^{-1}$ was used as reference for comparison with the others. Comparing the results obtained from the least refined to the most refined resolution, an average deviation of around 5\% was found in all the cases tested, which can be considered relatively low. From these results, the possibility to solve more complex problems using the LBL integration with a less accurate spectral resolution, without neglecting the computational reliability of the method, can be viable. These results are promising, since the greater the discretization of spectrum is, the greater the computational time spent in obtaining the solution becomes, especially in more complex engineering problems. As a new direction in the gas modeling research field, it can also be attempted to apply non-uniform discretization in the LBL solution, leading to considerable reduction in the computational time. 


\section{References}

1. Rothman LS, Gordon IE, Babikov Y, Barde A, Chris Benner D, Bernath PF, Birk M, Bizzocchi L, Boudon V, Brown LR, Campargue A, Chance K, Cohen EA, Coudert LH, Devi VM, Drouin BJ, Fayt A, Flaud J-M, Gamache RR, Harrison JJ, Hartmann J-M, Hill C, Hodges JT, Jacquemart D, Jolly A, Lamouroux J, Le Roy RJ, Li G, Long DA, Lyulin OM, Mackie CJ, Massie ST, Mikhailenko S, Müller HSP, Naumenko OV, Nikitin AV, Orphal J, Perevalov V, Perrin A, Polovtseva ER, Richard C, Smith MAH, Starikova E, Sung K, Tashkun S, Tennyson J, Toon GC, Tyuterev VLG, Wagner G (2013) The HITRAN 2012 molecular spectroscopic database. J Quant Spectrosc Radiat Transf 130:4-50

2. Rothman LS, Gordon IE, Barber RJ, Dothe H, Gamache RR, Goldman A, Perevalov VI, Tashkun SA, Tennyson J (2010) HITEMP, the high-temperature molecular spectroscopic database. J Quant Spectrosc Radiat Transf 111:2139-2150

3. Chu H, Liu F, Zhou H (2011) Calculations of gas thermal radiation transfer in one-dimensional planar enclosure using LBL and SNB models. Int J Heat Mass Transf 54:4736-4745

4. Rivière $P$, Soufiani A (2012) Updated band model parameters for $\mathrm{H}_{2} \mathrm{O}, \mathrm{CO}_{2}, \mathrm{CH}_{4}$ and $\mathrm{CO}$ radiation at high temperature. Int $\mathrm{J}$ Heat Mass Transf 55(13-14):3349-3358

5. Pearson JT, Webb BW, Solovjova VP, Ma J (2013) Updated correlation of the absorption line blackbody distribution function for $\mathrm{H}_{2} \mathrm{O}$ based on the HITEMP2010 database. J Quant Spectrosc Radiat Transf 128:10-17

6. Pearson JT, Webb BW, Solovjova VP, Ma J (2014) Efficient representation of the absorption line blackbody distribution function for $\mathrm{H}_{2} \mathrm{O}, \mathrm{CO}_{2}$, and $\mathrm{CO}$ at variable temperature, mole fraction, and total pressure. J Quant Spectrosc Radiat Transf 138:82-96

7. Pearson JT, Webb BW, Solovjova VP, Ma J (2014) Effect of total pressure on the absorption line blackbody distribution function and radiative transfer in $\mathrm{H}_{2} \mathrm{O}, \mathrm{CO}_{2}$, and CO. J Quant Spectrosc Radiat Transf 143:100-110

8. Liu F, Chu H, Zhou H, Smallwood GJ (2013) Evaluation of the absorption line blackbody distribution function of $\mathrm{CO}_{2}$ and $\mathrm{H}_{2} \mathrm{O}$ using the proper orthogonal decomposition and hyperbolic. J Quant Spectrosc Radiat Transf 128:27-33

9. Modest MF, Zhang H (2002) The full-spectrum correlated-k distribution for thermal radiation from molecular gas-particulates mixtures. J Heat Transf 124:30-38

10. Modest MF, Singh V (2005) Engineering correlations for full spectrum k-distribution of $\mathrm{H}_{2} \mathrm{O}$ from HITEMP spectroscopic databank. J Quant Spectrosc Radiat Transf 93:263-271

11. Cai J, Modest MF (2014) Improved full-spectrum k-distribution implementation for inhomogeneous media using a narrow-band database. J Quant Spectrosc Radiat Transf 141:65-72
12. Kangwanpongpan T, França FHR, da Silva RC, Schneider PS, Krautz HJ (2012) New correlations for the weighted-sum-ofgray-gases model in oxy-fuel conditions based on HITEMP 2010 database. Int J Heat Mass Transf 55:7419-7433

13. Dorigon LJ, Duciak G, Brittes R, Cassol F, Galarça M, França FHR (2013) WSGG Correlations based on HITEMP2010 for computation of termal radiation in non-isotheral, non-homogeneous $\mathrm{H}_{2} \mathrm{O} / \mathrm{CO}_{2}$ mixtures. Int J Heat Mass Transf 64:863-873

14. Cassol F, Brittes R, França FHR, Ezekoye OO (2014) Application of the weighted-sum-of-gray-gases model for media composed of arbitrary concentrations of $\mathrm{H}_{2} \mathrm{O}, \mathrm{CO}_{2}$ and soot. Int J Heat Mass Transf 79:796-806

15. Bordbar MH, Wecel G, Hyppänen T (2014) A line by line based weighted sum of gray gases model for inhomogeneous $\mathrm{CO}_{2}-\mathrm{H}_{2} \mathrm{O}$ mixture in oxy-fired combustion. Combust Flame 161:2435

16. Guo J, Li X, Huang X, Liu Z, Zheng C (2015) A full spectrumkdistribution based weighted-sum-of-gray-gases model for oxy-fuel combustion. Int J Heat Mass Transf 90:218-226

17. Brittes R, Centeno FR, Ziemniczak A, França FHR (2017) WSGG model correlations to compute non-gray radiation from carbon monoxide in combustion applications. J Heat Transfer 139:863-873

18. Centeno FR, Brittes R, Rodrigues LGP, Coelho FC, França FHR (2018) Evaluation of the WSGG model against line-by-line calculation of thermal radiation in a non-gray sooting medium representing an axisymmetric laminar jet flame. Int J Heat Mass Transf $124: 475-483$

19. Modest MF (2013) Radiative heat transfer, 3rd edn. Academic Press, New York

20. Chu H, Consalvi JL, Gu M, Liu F (2017) Calculations of radiative heat transfer in an axisymmetric jet diffusion flame at elevated pressures using different gas radiation models. J Quant Spectrosc Radiat Transf 197:12-25

21. Chu H, Gu M, Consalvi J, Liu F, Zhou H (2016) Effects of total pressure on non-grey gas radiation transfer in oxy-fuel combustion using the LBL, SNB, SNBCK, WSGG, and FSCK methods. J Quant Spectrosc Radiat Transf 172:24-35

22. Chu H, Ren F, Feng Y, Gu M, Zheng S (2017) A comprehensive evaluation of the non gray gas thermal radiation using the line-byline model in one- and two-dimensional enclosures. Appl Therm Eng 124:362-370

23. Siegel R, Howell J (2002) Thermal Radiation Heat transfer, 4th edn. Taylor and Francis, New York

Publisher's Note Springer Nature remains neutral with regard to jurisdictional claims in published maps and institutional affiliations. 